\title{
Separate Prefrontal-Subcortical Circuits Mediate Different Components of Risk-Based Decision Making
}

\author{
Jennifer R. St. Onge, ${ }^{1}$ Colin M. Stopper, ${ }^{1}$ Daniel S. Zahm, ${ }^{2}$ and Stan B. Floresco ${ }^{1}$ \\ ${ }^{1}$ Department of Psychology and Brain Research Center, University of British Columbia, Vancouver, British Columbia, V6T 1Z4 Canada and ${ }^{2}$ Department of \\ Pharmacological and Physiological Science, Saint Louis University School of Medicine, Saint Louis, Missouri 63104
}

Choosing between smaller, assured rewards or larger, uncertain ones requires reconciliation of competing biases toward more certain or riskier options. We used disconnection and neuroanatomical techniques to reveal that separate, yet interconnected, neural pathways linking the medial prefrontal cortex (PFC), the basolateral amygdala (BLA), and nucleus accumbens (NAc) contribute to these different decision biases in rats. Disrupting communication between the BLA and NAc revealed that this subcortical circuit biases choice toward larger, uncertain rewards on a probabilistic discounting task. In contrast, disconnections between the BLA and PFC increased choice of the Large/Risky option. PFC-NAc disconnections did not affect choice but did increase choice latencies and trial omissions. Neuroanatomical studies confirmed that projection pathways carrying axons from BLA-to-PFC transverse a distinctly different route relative to PFC-to-BLA pathways (via the ventrolateral amydalofugal pathway and ventromedial internal capsule, respectively). We exploited these dissociable axonal pathways to selectively disrupt bottom-up and top-down communication between the BLA and PFC. Subsequent disconnection studies revealed that disruption of top-down (but not bottom-up) information transfer between the medial PFC and BLA increased choice of the larger, riskier option, suggesting that this circuit facilitates tracking of actions and outcomes to temper urges for riskier rewards as they become less profitable. These findings provide novel insight into the dynamic competition between these cortical/ subcortical circuits that shape our decision biases and underlie conflicting urges when evaluating options that vary in terms of potential risks and rewards.

\section{Introduction}

We routinely have to evaluate the relative risks and rewards associated with different options, choosing between potentially more profitable, but uncertain outcomes, and safer, yet more modest, rewards, such as when managing an investment portfolio. Three key brain areas contribute to decision making about certain and uncertain rewards in humans, nonhuman primates, and rats: the prefrontal cortex (PFC), amygdala, and nucleus accumbens (NAc). Each of these regions are intimately interconnected: the PFC and amygdala share reciprocal connections (permitting both bottom-up and top-down processing of information), and the PFC and amygdala also send unidirectional projections to the NAc (McDonald, 1987, 1991a,b; Sesack et al., 1989; Brog et al., 1993; McDonald et al., 1996).

Across species, medial regions of the PFC (including the anterior cingulate) are thought to monitor choice information and

Received Nov. 8, 2011; revised Dec. 19, 2011; accepted Jan. 6, 2012.

Author contributions: J.R.S.O., D.S.Z., and S.B.F. designed research; J.R.S.O., C.M.S., and D.S.Z. performed research; J.R.S.O., C.M.S., D.S.Z., and S.B.F. analyzed data; J.R.S.O., C.M.S., D.S.Z., and S.B.F. wrote the paper.

This work was supported by grants from the Canadian Institutes of Health Research (MOP 89861) to S.B.F. and NIH NS-23805 to D.S.Z. S.B.F. is a Michael Smith Foundation for Health Research Senior Scholar and J.R.S.0. is the recipient of scholarships from the Natural Sciences and Engineering Research Council of Canada and the Michael Smith Foundation for Health Research. We thank Ken Parsely, Anita Y Cheng, Zachary M. Schwartz, and Erika Kirchnawy for providing excellent technical support.

Correspondence should be addressed to Dr. Stan B. Floresco, Department of Psychology and Brain Research Center, University of British Columbia, 2136 West Mall, Vancouver, B.C., V6T 124 Canada. E-mail: floresco@ psych.ubc.ca.

DOI:10.1523/JNEUROSCI.5625-11.2012

Copyright $\odot 2012$ the authors $\quad 0270-6474 / 12 / 322886-14 \$ 15.00 / 0$ adjust behavior in response to decision outcomes (Rogers et al., 2004; Marsh et al., 2007; St. Onge and Floresco, 2010), whereas the amygdala and ventral striatum play a key role in value representation (Knutson et al., 2005; Balleine and Killcross, 2006; Hampton et al., 2007; Ghods-Sharifi et al., 2009; Roesch et al., 2009; Pessoa, 2010). However, reliance on interpreting functions of different brain regions in isolation lacks external validity; determining the manner in which these regions interact to guide behavior is essential to understanding what drives us to make risky or safe decisions. Thus, the possibility remains that separate neural pathways within these brain circuits may differentially influence the direction of uncertain versus certain choices and regulate our ability to make decisions about different risks and rewards.

Functional imaging studies have provided indirect evidence that these regions do interact to facilitate decision making about probabilistic rewards. Functional connectivity between the anterior cingulate and the NAc has been observed when subjects are choosing high-risk compared with low-risk gambles (Cohen et al., 2005) and between the cingulate and amygdala when anticipating reward outcomes (Marsh et al., 2007). Given that it is difficult to directly test whether anatomical connectivity between structures is necessary for task performance in humans, rodent models of decision making provide a unique opportunity to directly disconnect specific pathways. Previous research in our laboratory using a probabilistic discounting task as a model of risk-based decision making in rats identified distinct roles for the basolateral amygdala (BLA), NAc, and the 
A

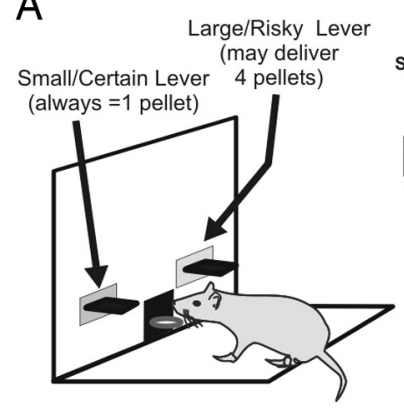

B

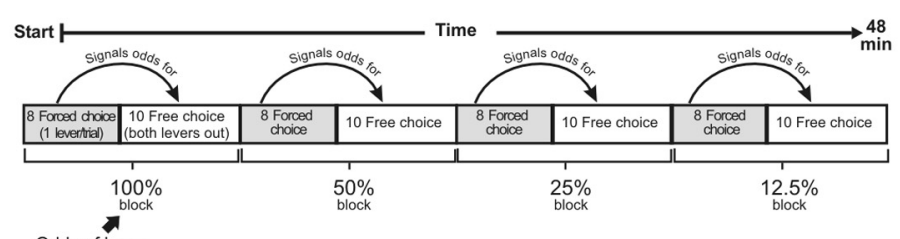

Odds of large

risky lever pres

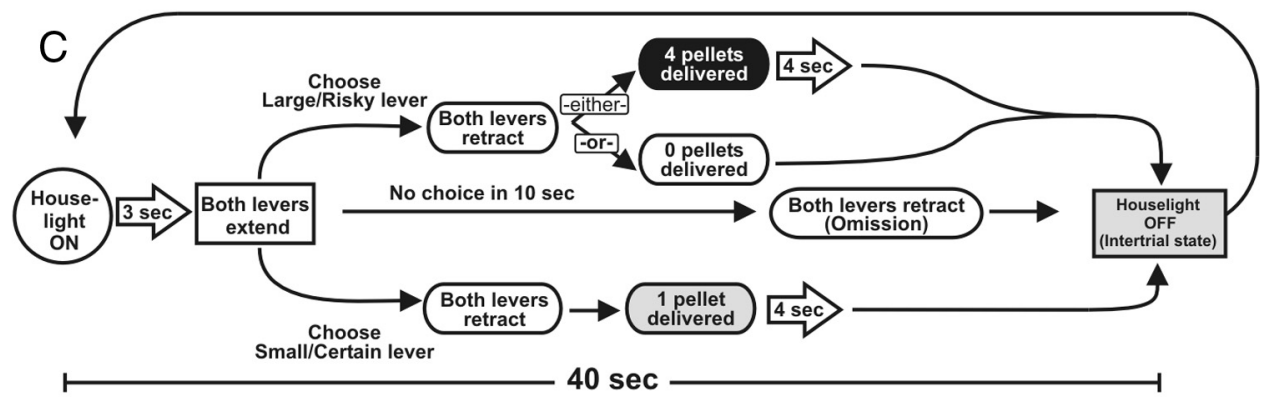

Figure 1. Probabilistic discounting task design. $A$, Cost/benefit contingencies associated with responding on either lever. $B$, Format of the sequence of forced and free choice trials within each probability block of a training session. C, Format of a single free-choice trial.

prelimbic medial PFC in biasing choice between uncertain versus certain rewards. Inactivation of either the BLA or NAc biases choice away from larger, uncertain rewards, whereas similar inactivation of the PFC induces a more complex deficit indicative of an inability to update decision policies (Ghods-Sharifi et al., 2009; St. Onge and Floresco, 2010; Stopper and Floresco, 2011). Thus, accumulating evidence suggests that a cortico-limbicstriatal circuit involving the medial PFC, BLA, and NAc may mediate decision making about probabilistic outcomes. What remain unclear are the specific routes of serial information transfer between these regions that facilitate decision making about different risks and rewards in a continuously changing environment. To address this, the present study used an asymmetrical disconnection approach to clarify the contribution of these different pathways to this aspect of decision making.

\section{Materials and Methods}

\section{Animals}

Adult male Long-Evans rats (Charles River Laboratories) weighing 275$300 \mathrm{~g}$ at the beginning of behavioral training were food restricted to $85-90 \%$ of their free-feeding weight for the remainder of the experiment. All testing was in accordance with the Canadian Council of Animal Care and the Animal Care Committee of the University of British Columbia.

\section{Apparatus}

Behavioral testing for all experiments described here was conducted in 12 operant chambers $(30.5 \times 24 \times 21 \mathrm{~cm}$; Med Associates $)$ enclosed in sound-attenuating boxes. The boxes were equipped with a fan to provide ventilation and to mask extraneous noise. Each chamber was fitted with two retractable levers, one located on each side of a central food receptacle where food reinforcement ( $45 \mathrm{mg}$; Bioserv) was delivered via a pellet dispenser. The chambers were illuminated by a single $100 \mathrm{~mA}$ house light located in the top-center of the wall opposite the levers. Four infrared photobeams were mounted on the sides of each chamber. Locomotor activity was indexed by the number of photobeam breaks that occurred during a session. All experimental data were recorded by an IBM personal computer connected to the chambers via an interface.

\section{Lever pressing training}

Our initial training protocols were identical to those described in our previous studies (Ghods-Sharifi et al., 2009; St. Onge and Floresco, 2009, 2010; Stopper and Floresco, 2011). On the day before their first exposure to the chambers, rats were given $\sim 25$ sugar reward pellets in their home cage. On the first day of training, two or three pellets were delivered into the food cup and crushed pellets were placed on a lever before the animal was placed in the chamber. Rats were first trained under a fixed-ratio 1 schedule to a criterion of 60 presses in $30 \mathrm{~min}$, first for one lever, and then repeated for the other lever (counterbalanced left/right between subjects). Rats were then trained on a simplified version of the full task. These 90 trial sessions began with the levers retracted and the operant chamber in darkness. Every $40 \mathrm{~s}$, a trial was initiated with the illumination of the houselight and the insertion of one of the two levers into the chamber. If the rat failed to respond on the lever within $10 \mathrm{~s}$, the lever was retracted, the chamber darkened, and the trial was scored as an omission. If the rat responded within $10 \mathrm{~s}$, the lever retracted and a single pellet was delivered with $50 \%$ probability. This procedure was used to familiarize the rats with the probabilistic nature of the full task. In every pair of trials, the left or right lever was presented once, and the order within the pair of trials was random. Rats were trained for $\sim 5-6 \mathrm{~d}$ to a criterion of 80 or more successful trials (i.e., $\leq 10$ omissions).

\section{Probabilistic discounting task}

Rats were trained daily 6-7 d/week. Each session began in darkness with both levers retracted (the intertrial state). An entire session consisted of 72 trials, and took $48 \mathrm{~min}$ to complete. Trials began every $40 \mathrm{~s}$ with illumination of the houselight and, $3 \mathrm{~s}$ later, insertion of one or both levers into the chamber (Fig. 1). For the forced choice trials that preceded each block of free choice trials, only one lever was presented ( 4 trials for each lever, randomized in pairs). Designation of the Large/Risky and Small/Certain lever was counterbalanced left/right across animals and remained consistent throughout training. Once a trial was initiated, no response within $10 \mathrm{~s}$ of lever presentation (omission) reset the chamber to the intertrial state (darkness, levers retracted) until the next trial. The probability of obtaining four pellets after pressing the Large/Risky lever decreased across blocks: it was initially $100 \%$, then 50\%, 25\%, and finally $12.5 \%$, for each successive block. Using these probabilities, selection of the Large/Risky lever would be advantageous in the first two blocks, and 
disadvantageous in the last block, whereas rats could obtain an equivalent number of food pellets after responding on either lever during the $25 \%$ block. Therefore, in the last three trial blocks of this task, selection of the larger reward option carried with it an inherent "risk" of not obtaining any reward. Latencies to initiate a choice and overall locomotor activity ( photobeam breaks) were also recorded.

Rats were trained until they displayed stable choice behavior, after which they were subjected to surgery. Stability was determined using data from three consecutive sessions that were analyzed with two-way repeated-measures ANOVAs with Day and Trial Block as factors. If there was no main effect of Day or Day $\times$ Trial Block interaction (at $p>0.1$ level), performance of the group was deemed stable.

\section{Reward magnitude discrimination task}

As we have done previously (Ghods-Sharifi et al., 2009; Stopper and Floresco, 2011), we determined a priori that if either ipsilateral inactivation or functional disconnection of the circuits connecting the medial PFC, BLA, or NAc decreased preference for the Large/Risky lever on the probabilistic discounting task, separate groups of animals would be trained and tested on a reward magnitude discrimination task to determine whether this effect was due to impairment in discriminating between reward magnitudes associated with the two levers. In these experiments, rats were also trained to press retractable levers as in the probabilistic discounting task, after which they were trained on the reward magnitude discrimination task. Here, rats chose between one lever that delivered one pellet and another that delivered four pellets. Both the small and large rewards were delivered immediately after a single response with $100 \%$ probability. A session consisted of four blocks of trials, with each block consisting of two forced-choice followed by 10 free-choice trials. After $\sim 10 \mathrm{~d}$ of training, rats typically displayed a strong preference for the four-pellet option. On the following day, rats received the first of three counterbalanced infusion test days (saline, ipsilateral inactivation, functional disconnection). Subsequent infusion test days were interspersed with 1-2 d of baseline training (no infusions).

\section{Disconnection design}

The logic underlying the use of asymmetrical disconnections inactivations to identify components of a functional neural circuit is based on the assumption that information is transferred serially from one structure to an efferent region on both sides of the brain in parallel. It further assumes that dysfunction will result from blockade of neural activity at the origin of a pathway in one hemisphere and the termination of the efferent pathway in the contralateral hemisphere. For example, if performance on a task is dependent on a serial connection linking the BLA to the NAc, then unilateral inactivation of the BLA would prevent the NAc in the ipsilateral hemisphere from gaining access to the information needed to perform the task. In the other hemisphere, information would be relayed from the BLA; however, suppression of neural activity within the NAc on this side of the brain would prevent it from processing incoming signals from the BLA. Thus, after this asymmetric disconnection, NAc neural activity that contributes to decision making processes would be compromised on both sides of the brain.

The disconnection design rests on two further assumptions. First, it assumes that ipsilateral inactivation of one or both structures in a circuit should not have as a disruptive effect on behavior, because the intact structures in the hemisphere should be able to at least partially compensate for the unilateral disruption in function. In the design, this assumption is controlled for by using ipsilateral inactivation of both structures in a circuit. Second, to be maximally effective, contralateral connections between two brain regions should be minimized. With respect to the pathways explored in the present study, projections from the BLA to both the NAc and PFC are primarily ipsilateral (McDonald, 1987, 1991a,b). However, the PFC sends both ipsilateral and contralateral descending projections to the BLA. To compensate for this, where we selectively disconnected descending PFC inputs to the BLA (using asymmetrical internal capsule/BLA inactivations), our surgical procedures included a transection of the corpus callosum in a region just caudal to the PFC, which is the region where axons from the ipsilateral PFC cross over and descend toward the contralateral BLA.

\section{Surgical, microinfusion, and testing procedures}

Rats were anesthetized with ketamine and xylazine (100 and $7 \mathrm{mg} / \mathrm{kg}$, respectively). Rats were implanted with two sets of bilateral 23 gauge stainless steel guide cannula using standard stereotaxic techniques. Five separate combinations of placements were used: (1) BLA [anteroposterior $(\mathrm{AP})=-3.1 \mathrm{~mm}$; medial-lateral $(\mathrm{ML})= \pm 5.2 \mathrm{~mm}$ from bregma; dorsoventral $(\mathrm{DV})=-6.5 \mathrm{~mm}$ from dura] and NAc $(\mathrm{AP}=+1.5 \mathrm{~mm}$; $\mathrm{ML}= \pm 1.4 \mathrm{~mm} ; \mathrm{DV}=-5.9 \mathrm{~mm}),(2) \mathrm{BLA}$ and medial PFC $(\mathrm{AP}=+3.4$ $\mathrm{mm} ; \mathrm{ML}= \pm 0.7 \mathrm{~mm} ; \mathrm{DV}=-2.8$ ), (3) PFC and NAc (same coordinates as above), (4) medial PFC and ventrolateral amydalofugal pathway (ascending $\mathrm{BLA} \rightarrow$ medial $\mathrm{PFC}$ pathway; $\mathrm{AP}=-0.5 \mathrm{~mm} ; \mathrm{ML}= \pm 5.0 \mathrm{~mm}$; $\mathrm{DV}=-5.3$ ), and (5) BLA and ventromedial internal capsule (descending $\mathrm{PFC} \rightarrow$ BLA pathway; $\mathrm{AP}=-1.5 \mathrm{~mm} ; \mathrm{ML}= \pm 2.4 \mathrm{~mm}$; $\mathrm{DV}=-6.3$ $\mathrm{mm}$ ). As described above, we also performed a $1 \mathrm{~mm}$ transection of the corpus callosum in the descending PFC-BLA pathway group. For PFCNAc preparations, the corpus callosum was transected by the guide cannula targeted at the NAc. We did not employ a similar transection for disconnection of ascending pathway because projections from BLA to PFC are primarily ipsilateral. Callosotomy alone typically has little to no effect on behavior (Floresco et al., 1999; Dunnett et al., 2005; Bezzina et al., 2008).

Rats were given at least 1 week to recover from surgery before being retrained for at least $5 \mathrm{~d}$. Once stable choice behavior was reestablished, they received their first microinfusion test day. On separate test days, rats received three counterbalanced infusions: (1) a saline infusion into both structures contralaterally (saline), (2) drug infusions in two regions in the same hemisphere (ipsilateral inactivation), and (3) drug infusions in two regions in opposite hemispheres (functional disconnection). The ipsilateral inactivation was used as a within-subjects control to determine whether mass action effects may occur when we simultaneously inactivate two brain regions. The order of treatments and the hemispheres that received ipsilateral/contralateral infusions were counterbalanced across animals. Following the first infusion test day, rats received 1-2 baseline training days (no infusion) until an individual rat's choice of the Large/ Risky lever deviated by $<15 \%$ from its preinfusion baseline. On the following day, a rat received a second counterbalanced infusion, followed by another training day, and lastly, the final infusion.

\section{Histology}

After completion of behavioral testing, rats were killed in a carbon dioxide chamber. Brains were removed and fixed in a $4 \%$ formalin solution. The brains were frozen and sliced in $50 \mu \mathrm{m}$ sections before being mounted and stained with Cresyl Violet. Placements were verified with reference to a neuroanatomical atlas (Paxinos and Watson, 1998). Data from rats whose placements were outside the borders of the medial PFC, NAc, BLA, or the targeted axon fiber bundles along the ventrolateral edge of the corpus callosum and the ventromedial internal capsule were removed from the analysis.

\section{Data analysis}

The primary dependent measure of interest for the probabilistic discounting task was the proportion of choices directed toward the Large/ Risky lever for each block of free-choice trials, factoring in trial omissions. For each block, this was calculated by dividing the number of choices of the Large/Risky lever by the total number of successful trials. Choice data were analyzed using either one- or two-way within-subjects ANOVAs, with Treatment and Trial Block as within-subjects factors. Multiple comparisons were made with Dunnett's or Newman-Keuls tests as appropriate. In each of these analyses, the effect of Trial Block was always significant $(p<0.001)$ and will not be mentioned further. Locomotor activity (i.e., photobeam breaks), response latency, and the number of trial omissions were analyzed with one-way repeated-measures ANOVAs.

If we observed a significant change in preference for the Large/Risky lever on the probabilistic discounting task, we conducted a supplementary analysis to obtain further insight into how these treatments affected patterns of choice and resulting alterations in discounting. Specifically, this choice-by-choice analysis was designed to identify whether changes in behavior were due to alterations in the likelihood of choosing the risky 
lever after obtaining the larger reward (win-stay performance) or alterations in negative feedback sensitivity (lose-shift performance) (St. Onge et al., 2011; Stopper and Floresco, 2011). Animals' choices during the task were analyzed according to the outcome of each preceding free-choice trial (reward or nonreward) and expressed as a ratio. The proportion of win-stay trials was calculated from the number of times the rat chose the Large/Risky lever after choosing the risky option on the preceding trial and obtaining the large reward (a win), divided by the total number of free-choice trials where the rat obtained the larger reward. Conversely, lose-shift performance was calculated from the number of times rats shifted choice to the Small/Certain lever after choosing the risky option on the preceding trial and were not rewarded (a loss), divided by the total number of free-choice trials resulting in a loss. This analysis was conducted for all trials across the four blocks. We could not conduct a block-by block analysis of these data because there were many instances where rats did not obtain the large reward at all during the latter blocks. Changes in win-stay performance were used as an index of the impact that obtaining the large, risky reward had on subsequent choice behavior, whereas changes in lose-shift performance served as an index of negative feedback sensitivity.

\section{Anatomical tract tracing studies tracer injections}

A separate neuroanatomical study was undertaken to determine the specific routes that projection axons from the BLA $\rightarrow$ PFC and PFC $\rightarrow$ BLA take through the brain. Seven rats were deeply anesthetized by intraperitoneal injections of a mixture of ketamine $(72 \mathrm{mg} / \mathrm{kg})$ and xylazine $(11.2$ $\mathrm{mg} / \mathrm{kg}$ ). The anesthetic was injected as a mixture consisting of $45 \%$ ketamine $(100 \mathrm{mg} / \mathrm{ml}), 35 \%$ xylazine $(20 \mathrm{mg} / \mathrm{ml})$, and $20 \%$ saline at a dose of $0.16 \mathrm{ml} / 100 \mathrm{~g}$ of body weight. Several minutes later, rats were placed in a Kopf stereotaxic instrument. Skulls were exposed and bore holes were created to allow selected brain structures to be targeted by filamentcontaining borosilicate glass pipettes $(\mathrm{OD}, 1.0 \mathrm{~mm}$ ) pulled to tip diameters of 10 or $25 \mu \mathrm{m}$, and containing an anterograde tracer, either Phaseolus vulgaris leucoagglutinin (PHA-L; Vector, $2.5 \%$ in $0.01 \mathrm{M}$ phosphate buffer) or biotinylated dextran amine (BDA, Invitrogen; $10 \%$ in $0.01 \mathrm{~m}$ phosphate buffer). A silver wire inserted into the pipettes contacted the solution containing the tracer, which was ejected into the brain substance using positive current pulses ( $7 \mathrm{~s}$ on, $7 \mathrm{~s}$ off, for $15 \mathrm{~min}$ ) of 4 $\mu \mathrm{A}$. After surgery, rats were kept warm until they awakened.

One case with BDA injected into the BLA was plotted and is shown in Figure 5. Two cases with injections of PHA-L in the prelimbic cortex were used and one of these is shown in Figure 6. BDA is transported retrogradely from some injection sites and thus can occasionally produce spurious anterograde labeling from back-filled neurons. Although backfilled neurons were not identified in the case with injection of BDA into the BLA, an additional four cases were prepared with PHA-L injections into the BLA. No differences between the case with BDA compared with PHA-L injection into the BLA were detected.

Fixation of brains and immunocytochemistry. Ten days after PHA-L injections and $5 \mathrm{~d}$ after BDA injections, the rats were deeply anesthetized (as above) and perfused transaortically, first with $0.01 \mathrm{M}$ Sorensen's phosphate buffer (SPB; pH 7.4) containing $0.9 \%$ sodium chloride and $2.5 \%$ sucrose, followed by $0.1 \mathrm{M} \mathrm{SPB}$, pH 7.4, containing $4 \%$ paraformaldehyde and $2.5 \%$ sucrose. The brains were removed, postfixed, infiltrated with $25 \%$ sucrose, sectioned, and frozen at $50 \mu \mathrm{m}$. Five adjacent series of sections were collected, thus reflecting the structure of the entire brain from frontal pole to caudal medulla in sections spaced at intervals of 250 $\mu \mathrm{m}$. Each series of sections was stored in a separate glass vial at $-20^{\circ} \mathrm{C}$ in a cryoprotectant consisting of SPB containing $30 \%$ sucrose (by weight) and $30 \%$ ethylene glycol (by volume).

For PHA-L, a series of sections from each case was immersed in SPB containing $0.1 \%$ Triton X-100 (SPB-t) and polyclonal antibodies raised against PHA-L made in goat used at a dilution of 1:10,000. The following day, after thorough rinsing in SPB-t, the sections were immersed for an hour in SPB-t containing biotinylated antibodies made in goat at a dilution of 1:200 (Jackson ImmunoResearch Laboratories). Afterward, the sections were rinsed in SPB and then immersed in SPB containing avidinbiotin-peroxidase complex (ABC; Vector Laboratories) at a dilution of 1:200 for an hour. Cases with BDA injections were pretreated by immer- sion in $1 \%$ aqueous sodium borohydride for 15 min followed by thorough rinsing and then placed immediately in SPB-t containing $\mathrm{ABC}$ reagents at a dilution of 1:200. After additional thorough rinsing in SPB, reaction products for PHA-L and BDA processed sections were generated by immersing the sections for $20-30 \mathrm{~min}$ in $0.05 \mathrm{M} \mathrm{SPB}, \mathrm{pH} 7.4$, containing $0.05 \% \mathrm{DAB}, 0.04 \%$ ammonium chloride, $0.2 \% \beta$-D-glucose, and $0.0004 \%$ glucose oxidase, which generates an insoluble brown reaction product. The sections were then mounted on glass slides and allowed to dry. The reaction product was intensified by immersion of the slides first in $4 \%$ osmium tetroxide in distilled $\mathrm{H}_{2} \mathrm{O}$, then in $0.1 \%$ thiocarbohydrazide, and again in the osmium solution with running tap water rinses between. The sections were again dried and coverslipped with Permount (Fisher Scientific).

Maps. Anterograde labeling was plotted in representative frontal sections throughout the CNS (excluding spinal cord) with the $10 \times$ or $20 \times$ objective under brightfield optics (Olympus BX51) with the aid of the Neurolucida dedicated hardware-software platform (MBF Bioscience). Tracing was mapped in a manner such that areas of the brain containing dense axonal varicosities, i.e., puncta, were represented as a fine stippling. The smooth, nonvaricose, mainly nonsynaptic parts of labeled axons were directly traced. The plots were exported to Adobe Illustrator (version CS2) as .emf files and the maps were finished and labeling added in Illustrator.

\section{Results}

\section{Effect of functional disconnection of the BLA-NAc pathway on probabilistic discounting}

Disruption of neural activity in both the NAc and BLA reduces preference for large, risky options (Ghods-Sharifi et al., 2009; Stopper and Floresco, 2011). Therefore, our first aim was to assess whether these two regions form a functional neural circuit that normally biases choice toward larger, risky rewards. Initially, 16 rats were trained for $\sim 32 \mathrm{~d}$ on the probabilistic discounting task before showing stable choice behavior and proceeding to microinfusion test days. Two died during surgery and the data from three others was eliminated due to inaccurate placements, resulting in a final $n$ of 11 rats in this group. Functional disconnection of this circuit significantly decreased choice of the Large/ Risky lever compared with saline $(n=11$; Fig. $2 A, B)$. Ipsilateral inactivation also reduced choice of this option. These impressions were confirmed by analysis of the choice data, which showed that these treatments decreased choice of the Large/Risky lever compared with saline treatment $\left(F_{(1,10)}=11.46, p=0.01\right)$. Furthermore, a direct comparison of the effect of BLA-NAc disconnection to saline also revealed a significant decrease in Large/ Risky lever choice $\left(F_{(1,10)}=6.60, p=0.03\right)$, whereas a similar analysis on the effects of ipsilateral inactivation did not yield a statistically reliable effect $\left(F_{(1,10)}=3.25, p=0.10\right)$.

The effect of ipsilateral inactivation on choice was driven primarily by four of the 11 rats. Visual inspection of the individual data showed that these rats clearly differed from the rest of the group, displaying $>20 \%$ decrease in choice of the Large/Risky lever across trial blocks after ipsilateral inactivation relative to saline infusions. Thus, in these rats, disconnection of the BLANAc pathway in only one hemisphere was as sufficient to alter choice as robustly as asymmetrical infusions. In comparison, the remaining seven rats showed almost no difference in overall choice of the risky option after saline versus ipsilateral inactivation infusions ( $<2 \%$ decrease). When we conducted a supplementary analysis on the choice data obtained from these other seven rats that showed comparable patterns of choice after ipsilateral inactivation and saline treatments, we again observed that asymmetrical inactivation of the BLA/NAc significantly reduced preference for the Large/Risky option $\left(F_{(2,12)}=4.25, p=0.04\right.$; Fig. $2 A$, inset). This latter finding suggests that, at least for the 
majority of animals that were unaffected by ipsilateral inactivations, the effects of asymmetrical BLA-NAc disconnections on choice were specifically attributable to a disruption of information transfer between these two regions. This decrease in risky choice did not appear to be the result of selective alterations in reward or negative feedback sensitivity. A choice-bychoice analysis of win-stay performance (i.e; choosing the Large/Risky option after obtaining the larger reward on the previous trial) showed that although both ipsilateral inactivation and functional disconnection tended to decrease win-stay performance compared with saline treatment, this effect was not statistically significant $\left(F_{(2,20)}=2.05\right.$, n.s.; Fig. $\left.2 C\right)$. Similarly, inactivation treatments tended to increase lose-shift tendencies (i.e.; selecting the safe option after a risky choice and loss), but the effect failed to reach significance $\left(F_{(2,20)}=\right.$ 1.99, n.s.; Fig. 2C). Similar results were obtained when we analyzed the data from the subset of rats that showed comparable performance on saline and ipsilateral inactivation test days (win-stay: $F_{(2,12)}=2.29$, n.s.; lose-shift: $F_{(2,12)}=0.22$, n.s.; data not shown).

Functional disconnection and ipsilateral inactivation of the BLA-NAc pathway reduced preference for the larger, uncertain reward during the discounting task. To assess whether these effects were the result of a general disruption in discriminating between different rewards magnitudes, we conducted another experiment, wherein a separate group of rats was trained to choose between two levers that delivered either one or four pellets, both with $100 \%$ probability. Initially, eight rats were used for this experiment and required $9 \mathrm{~d}$ of training before showing stable choice of the large reward lever. One animal died following surgery, resulting in a final $n$ of seven rats in this group. Under these conditions, ipsilateral inactivations or functional disconnection of the BLA-NAc pathway did not alter preference for the larger reward $\left(F_{(2,12)}=0.28\right.$, n.s.; Fig. $2 D)$. Moreover, inactivation treatments did not affect response latencies, locomotion, or trial omissions (all Fs $<2.43$, all $p s>0.12$; Table 1), indicating that the effects on decision making were unlikely to be the result of disruptions in motivational or motor processes or a reduction in general preference for larger rewards. Note that BLA-NAc disconnections decreased choice of the Large/Risky lever on the $100 \%$ block of the discounting task, even though similar disconnections did not affect decision making during the reward magnitude discrimination task. A potential explanation for this discrepancy may be that for those animals trained on the magnitude discrimination, the relative value of the larger reward
-BLA-NAc disconnection-

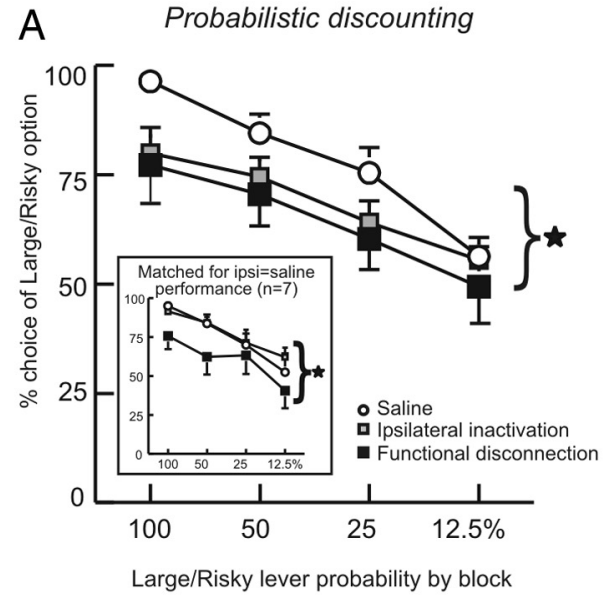

C $\begin{gathered}\text { - Reward/Negative Feedback Sensitivity- } \\ \text { (BLA } \rightarrow \text { Nac Disconnections) }\end{gathered}$

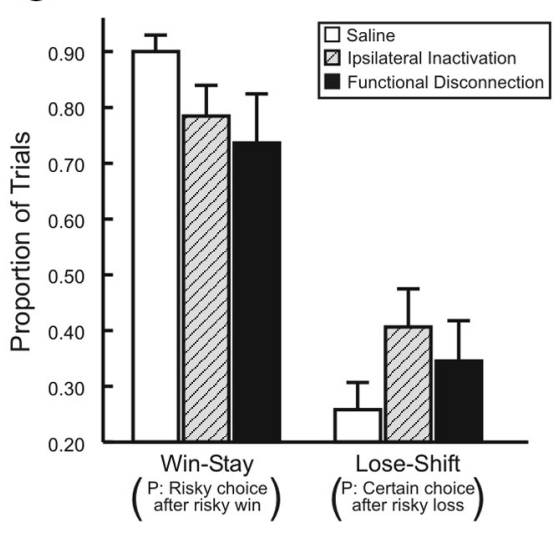

B

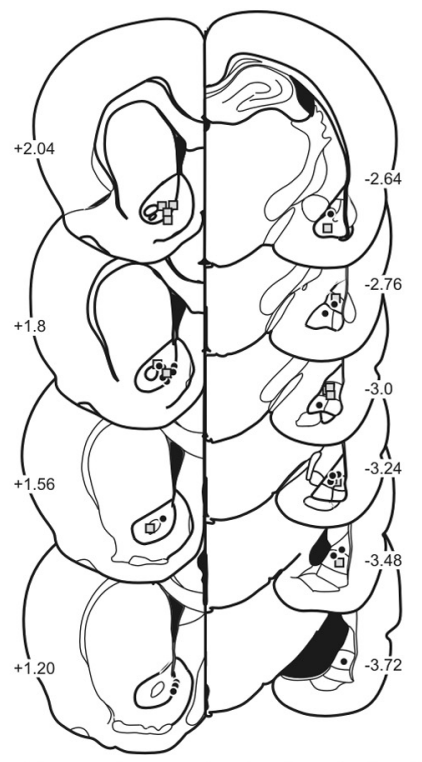

Deward magnitude discrimination

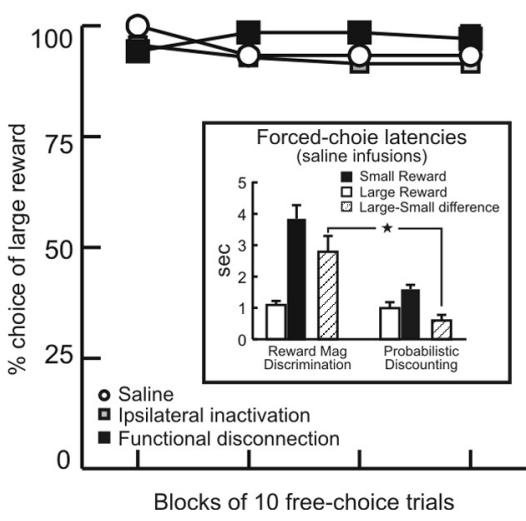

Figure 2. Disconnection of the BLA-NAC pathway reduces choice of the Large/Risky option, but does not affect reward magnitude discrimination. $A$, Percentage choice of the Large/Risky lever following disconnection of the NAc and BLA and control treatments across four blocks of free-choice trials. Symbols represent mean, error bars are SEM. Black star denotes $p<0.05$ for the average choice from ipsilateral/disconnection versus saline. Inset shows data from a subset of rats that did not show a decrease in risky choice after ipsilateral inactivation ( $n=7$ ), yet still showed a decrease in risky choice following functional disconnection (star denotes $p<0.05$ ). $B$, Schematic of sections of the rat brain showing location of acceptable infusions in the NAc and BLA for rats in discounting (circles) and magnitude discrimination (squares) experiments. Numbers correspond to millimeters from bregma. Figure represents the disconnection procedure for clarity; hemispheres of infusions were counterbalanced across rats. C, Win-stay/ lose-shift ratios following saline infusions, ipsilateral, and functional disconnection (asymmetrical inactivation) of the BLA-NAC pathway. Win-stay values are displayed as the proportion of choices on the Large/Risky lever following a rewarded risky choice on the preceding trial. Lose-shift values are displayed as the proportion of choices on the Small/Certain lever following unrewarded risky choice on the preceding trial. Neither of these measures were significantly altered by BLA-NAc disconnections. D, Disrupting communication in this pathway had no effect on preference for larger versus smaller rewards on a simpler reward magnitude discrimination. Inset shows response latencies to press the large or small reward lever on forced choice trials after saline infusions for rats trained on the reward magnitude discrimination or the probabilistic discounting task. Rats trained on the latter showed a smaller difference between latencies to press the larger versus smaller reward lever compared with those trained on the simpler magnitude discrimination (star denotes $p<0.01$ ).

is always higher than the smaller reward. In comparison, rats trained on probability discounting consistently experienced decreases in value of the large reward option over a session. Therefore, rats trained on the discounting task come into a training session with previous knowledge about the changing value of the large reward option, which may diminish their representation of the relative value discrepancy between the larger versus smaller reward option. In support of this theory, we observed that rats 


\begin{tabular}{|c|c|c|c|}
\hline Experiment & Saline & $\begin{array}{l}\text { Ipsilateral } \\
\text { inactivation }\end{array}$ & $\begin{array}{l}\text { Functional } \\
\text { disconnection }\end{array}$ \\
\hline \multicolumn{4}{|c|}{ BLA-NAc disconnection (discounting) } \\
\hline Response latency & $0.75(0.13)$ & $1.33(0.30)$ & $1.14(0.24)$ \\
\hline Trial omissions & $0.27(0.19)$ & $5.54(2.12)$ & $3.45(2.10)$ \\
\hline Locomotion & $1745(202)$ & 1517 (146) & 1428 (151) \\
\hline \multicolumn{4}{|c|}{$\begin{array}{l}\text { BLA-NAc disconnection (magnitude } \\
\text { discrimination) }\end{array}$} \\
\hline Response latency & $0.83(0.12)$ & $0.85(0.09)$ & $0.68(0.07)$ \\
\hline Trial omissions & $0(0)$ & $0.14(0.14)$ & $0(0)$ \\
\hline Locomotion & 1342 (151) & 1227 (204) & 1195 (138) \\
\hline \multicolumn{4}{|l|}{ PFC-BLA disconnection } \\
\hline Response latency & $0.55(0.04)$ & $0.89(0.15)$ & $0.86(0.11)$ \\
\hline Trial omissions & $0.18(0.12)$ & $1.09(0.55)$ & $2.27(0.89)$ \\
\hline Locomotion & $1513(143)$ & $1418(126)$ & $1418(162)$ \\
\hline \multicolumn{4}{|c|}{ BLA-PFC ascending pathway disconnection } \\
\hline Response latency & $0.81(0.08)$ & $0.76(0.13)$ & $0.72(0.10)$ \\
\hline Trial omissions & $2.9(2.35)$ & $0.5(0.31)$ & $2.5(1.97)$ \\
\hline Locomotion & 1736 (222) & 1781 (191) & $1693(175)$ \\
\hline \multicolumn{4}{|c|}{ PFC-BLA descending pathway disconnection } \\
\hline Response latency & $0.49(0.04)$ & $0.83(0.28)$ & $0.65(0.11)$ \\
\hline Trial omissions & $0(0)$ & $2.55(2.15)$ & $2.27(1.23)$ \\
\hline Locomotion & $1525(165)$ & $1362(165)$ & $1518(178)$ \\
\hline \multicolumn{4}{|l|}{ PFC-NAc disconnection } \\
\hline Locomotion & $1142(92)$ & 1007 (108) & $778(80)^{*}$ \\
\hline
\end{tabular}

trained on the reward magnitude discrimination showed substantially greater response latencies when forced to choose the smaller versus the larger reward lever under control conditions (Fig. $2 D$, inset). In comparison, for rats trained on the discounting task, this difference during the forced choice trials in the $100 \%$ probability block was significantly muted (Task $\times$ Reward Lever interaction, $F_{(1,16)}=21.78, p<0.001$; Fig. $2 D$, inset). A similar difference between groups was observed after inactivation treatments $\left(F_{(1,16)}=11.36, p<0.01\right.$; data not shown $)$. This suggests that rats trained on the two tasks differed in how they perceived the relative incentive value of the larger versus the smaller reward option. Rats trained on the simpler magnitude discrimination appear to view the one-pellet option as substantially inferior to the four-pellet option (based on their response latencies), whereas for those in the discounting group, this discrepancy was significantly smaller. This may explain why choice biases toward this option early in a discounting session were more sensitive to disruption of this circuitry. Collectively, these data show that neural activity within this subcortical amygdalar-ventral striatal pathway plays a key role in biasing choice behavior toward options yielding larger rewards that may be risky, but may also be more profitable when reward probabilities are high.

\section{Effect of functional disconnection of the medial PFC-BLA pathway on probabilistic discounting}

In rats, inactivation of the medial prelimbic PFC (homologous to Brodmann's area 32 of the anterior cingulate) increases choice of a larger, risky option when the odds of obtaining a larger reward are initially favorable and subsequently decrease, suggesting that this region updates and modifies choice biases when reward probabilities change (St. Onge and Floresco, 2010). Notably, this effect is opposite to that caused by inactivation of BLA (GhodsSharifi et al., 2009), a region that shares reciprocal connections with the PFC (Sesack et al., 1989; McDonald, 1991a; McDonald et al., 1996). Despite the seemingly contrasting roles these regions play in guiding decision making, we were particularly interested in assessing whether the PFC and BLA form a functional neural circuit that can influence the direction of choice on this task.

Initially, 16 rats were trained for $31 \mathrm{~d}$ before showing stable choice behavior and proceeding to microinfusion test days. One animal died following surgery, one rat had a compromised test day, and three animals had inaccurate placements, resulting in a final $n$ of 11 rats in this group. We observed that disconnection of the BLA-PFC pathway significantly increased choice of the Large/Risky lever (Fig. $3 A$ ), an effect similar to bilateral PFC inactivation (St. Onge and Floresco, 2010), but opposite to that observed after BLA inactivation (Ghods-Sharifi et al., 2009) or BLA-NAc disconnections (Fig. 2A). Analysis of the choice data revealed a significant Treatment $\times$ Block interaction $\left(F_{(6,60)}=\right.$ $2.24, p=0.05)$. Simple main effects analyses further revealed that asymmetrical PFC-BLA disconnections increased choice of the Large/Risky lever in the last two trial blocks relative to saline and relative to ipsilateral inactivation in the $12.5 \%$ block (Newman-Keuls, $p<0.05$; Fig. $3 A$ ). In contrast, ipsilateral inactivation did not affect choice relative to saline. Locomotion was not affected by inactivation treatments $\left(F_{(2,20)}=0.32\right.$, n.s. $)$, although these treatments did cause a slight increase in response latencies $\left(F_{(2,20)}=3.16, p=0.06\right)$ and trial omissions $\left(F_{(2,20)}=2.75, p=0.08\right.$; Table 1$)$.

A supplemental analysis of win-stay and lose-shift tendencies provided additional insight into the specific processes affected by disconnection of the PFC-BLA pathway. Win-stay performance was unaffected by these treatments $\left(F_{(1,10)}=\right.$ 0.04 , n.s.; Fig. $3 B$ ), suggesting that increased choice of the Large/Risky lever was not attributable to an enhanced tendency to choose this option after obtaining the larger reward on a preceding trial. However, BLA-PFC disconnection did reduce lose-shift tendencies relative to saline (Fig. $3 B$ ). Thus, under control conditions, when animals selected the Large/ Risky option and were not rewarded, they shifted to the Small/ Certain option on $\sim 35 \%$ of subsequent trials. However, following PFC-BLA disconnection, rats were much less likely to choose conservatively after not being rewarded for a risky choice, and analysis of these data showed a strong trend that approached statistical significance $\left(F_{(1,10)}=4.51, p=\right.$ $0.059)$. From these data, we infer that BLA-medial PFC circuitry enables adjustments in decision making when reward probabilities change, and appears to do so more so by mitigating choice behavior in response to negative feedback (i.e.; reward omissions).

\section{Effect of functional disconnection of the medial PFC-NAc pathway on probabilistic discounting}

In contrast to the above-mentioned findings, disconnection of outputs from the PFC to NAc did not affect probabilistic discounting $\left(n=12\right.$; main effect: $F_{(2,22)}=2.40, p=0.11$; interaction: $F_{(6,66)}=0.60, p=0.73$; Fig. $4 A$ ). However, these treatments increased trial omissions, choice latencies, and reduced locomotion (all Fs $>3.92$, all $p s<0.05$, Fig. $4 B, C$, Table 1 ). These latter results suggest that although communication between the PFC and ventral striatum does not appear to influence the manner in which choice of larger, probabilistic rewards are discounted, disrupting communication in this pathway does impair attention/ vigilance aspects of task performance and increases deliberation times. 


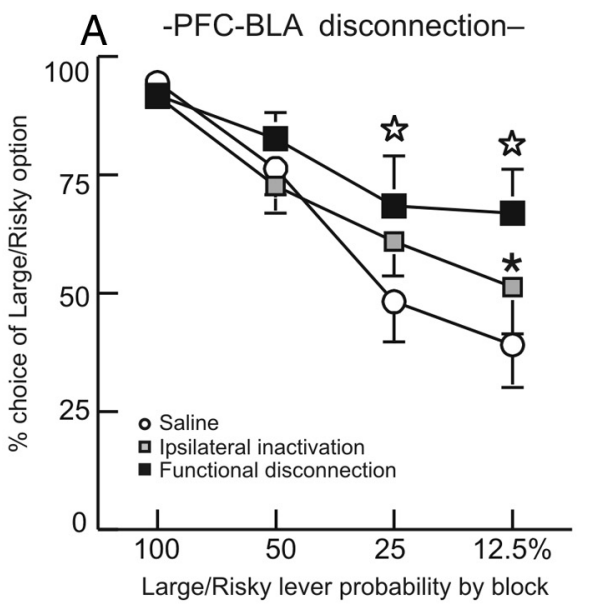

B - Reward/Negative Feedback Sensitivity-
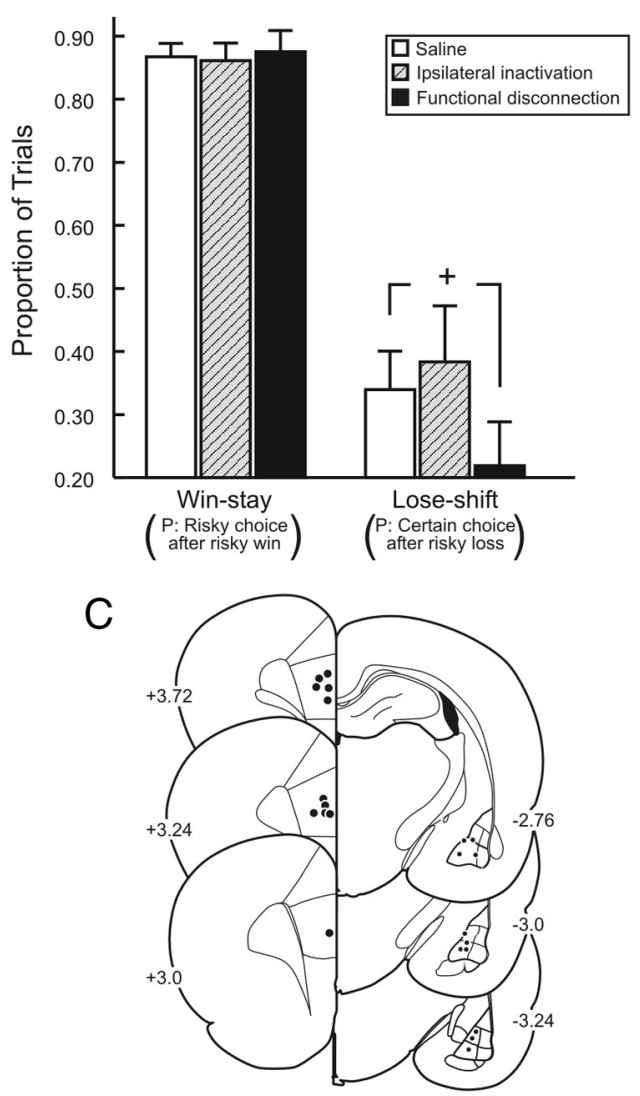

Figure 3. Disconnection of the medial PFC-BLA pathway increased choice of Large/Risky option. A, Choice for the Large/Risky lever following medial PFC-BLA disconnections and control treatments. Open star, $p<0.05$ saline versus functional disconnection; ${ }^{*} p<0.05$ (ipsilateral vs disconnection). $\boldsymbol{B}$, Win-stay/lose-shift data. Disconnection of the medial PFC-BLA pathway decreased negative feedback sensitivity, reducing the tendency to choose the certain option after a nonrewarded risky choice ${ }^{+} p=0.059$. C, Acceptable location of infusions through the rostral-caudal extent of the medial PFC and BLA.

\section{Anatomical dissociation of ascending $\mathrm{BLA} \rightarrow \mathrm{PFC}$ and descending $\mathrm{PFC} \rightarrow$ BLA axonal pathways}

The findings described above confirm that serial information transfer between the PFC and BLA is of critical importance to adjusting decision-making biases in response to changes in reward probability. However, what remained to be resolved was the directionality of the communication between these two regions. As the BLA and PFC share reciprocal connections, disconnection of these regions would be expected to disrupt both bottom-up transfer of reward value information from BLA to PFC and topdown signals from PFC to BLA.

The proposition that ascending and descending axonal projections connecting the BLA and medial PFC use anatomically distinct pathways has not been addressed experimentally to our knowledge, nor have several existing reports on the efferent connections of the medial PFC (Sesack et al., 1989; Groenewegen et al., 1990; Bacon et al., 1996) and BLA (Krettek and Price, 1977; McDonald, 1991a; Orozco-Cabal et al., 2006) included careful evaluation of the pathways that such efferents use to reach various target structures. Thus, for the purposes of the present study, we carefully analyzed material from normal rat brain injected with the anterogradely transported axonal tracers PHA-L or BDA into the medial PFC and BLA.

We observed that, for the BLA and consistent with previous reports (Krettek and Price, 1977), ascending projections from the BLA traverse three pathways: the stria terminalis, ventral amygdalofugal pathway, and external capsule (Fig. 5). BLA axons projecting via the stria terminalis and ventral amygdalofugal pathway arborize abundantly among the bed nucleus of stria terminalis, central amygdaloid nucleus, anterior amygdaloid area, sublenticular extended amgydala, interstitial nucleus of the posterior limb of the anterior commissure, and related basal forebrain and hypothalamic structures, suggesting that their distributions are mainly among those structures (Fig. 5D-I). In contrast, the pathway occupying the external capsule and adjacent deep cortical layers ascends in minimally arborized fashion before nearing the lateral and medial prefrontal cortical targets of the BLA, suggesting that these axons target prefrontal regions primarily, with minimal branching off to other intervening regions. Approaching the prefrontal targets, highly branched and varicose axons diverge medialward into and around the rostral pole of the accumbens toward the medial prelimibc PFC (Fig. 5A-C), which numerous labeled axons enter in abundantly arborized fashion. Based on these findings, we decided to inactivate the pathway ascending in the external capsule and adjacent cortex at the level circled in Figure 5D. Based on previous estimates of the functional spread of inactivation induced by infusion of local anaethestics in cortex ( $\sim 1 \mathrm{~mm}$ diameter) (Tehovnik and Sommer, 1997), it is likely suppression of neural activity induced by bupivacaine infusions would be relatively circumscribed to this region, with minimal spill over into other cortical areas.

Projections from the medial PFC mainly descend in two pathways (Fig. $6 B, C$ ). Most medial PFC efferents enter fiber bundles in the medial part of the rostral caudate-putamen (Fig. $6 B, D$ ), or cross to the opposite hemisphere in the corpus callosum (Fig. 6B) before joining the internal capsule. At the level of the sublenticular region, numerous labeled fibers leave the internal capsule and diverge lateralward across the sublenticular region toward to the amygdala (Fig. $6 F-H$ ). A second pathway out of the medial PFC comprises numerous fibers unconstrained within fascicles that pass through and around the rostral parts of the accumbens and caudate-putamen toward the lateral PFC. Beyond the insular cortex, this pathway occupies the deep cortex beneath the rhinal sulcus. It diminishes to negligible numbers of fibers before reaching the amygdala, suggesting that it is not a major pathway to that structure. Based on these considerations, we decided to inactivate the pathway confined to the internal capsule at the level circled in Figure $6 G$, insofar as just caudal to there, many fibers emerge singly and in small fascicles to cross the sublenticular region lateralward toward the amygdala, where many of them coalesce in a dense, varicose plexus in the BLA (Fig. 6I). 

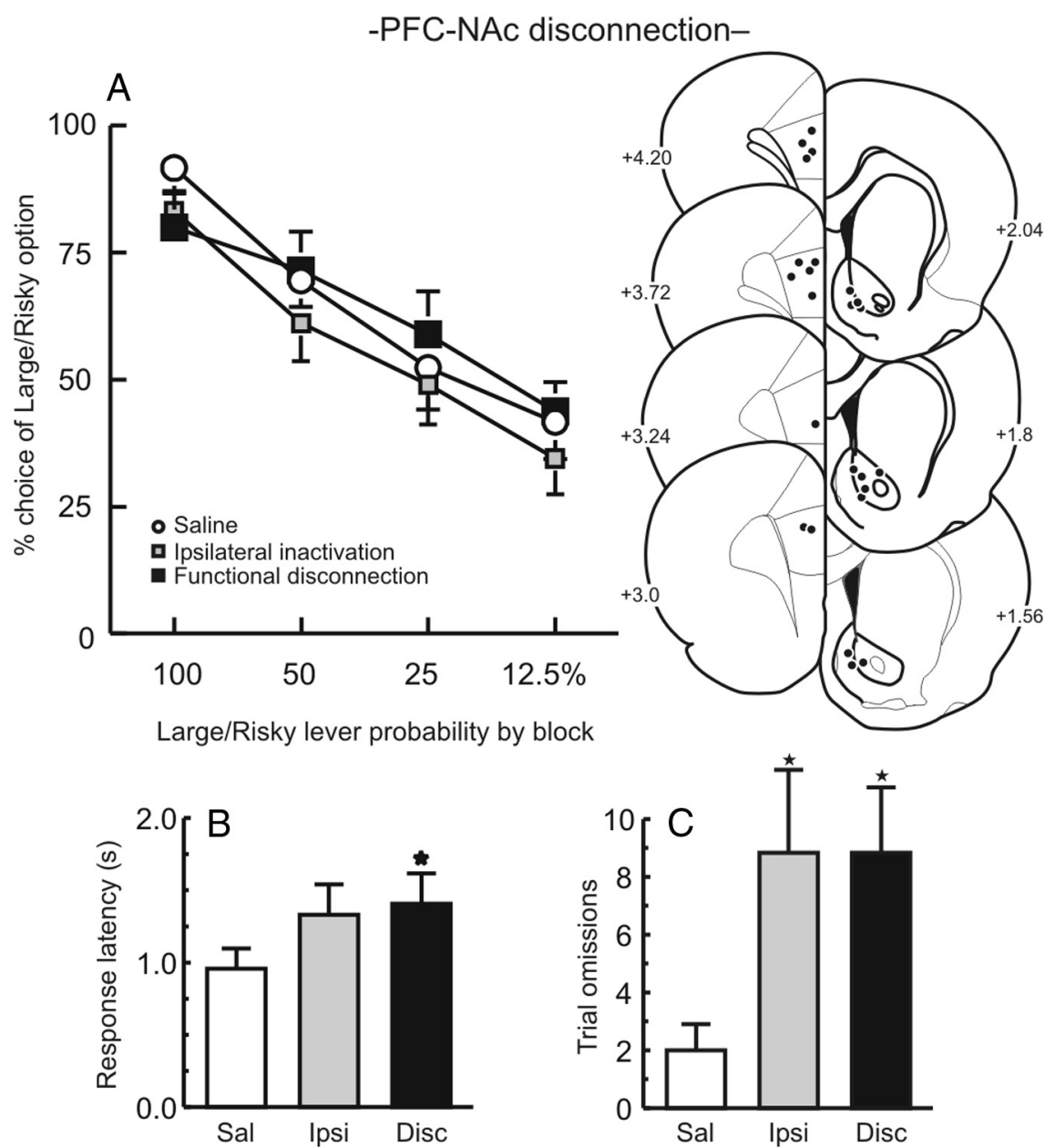

Figure 4. Disconnecting the medial PFC and NAc does not affect probabilistic discounting. $\boldsymbol{A}$, Left, Choice for the Large/Risky lever following medial PFC-NAc disconnections and control treatments. Right, Acceptable location of infusions through the rostral-caudal extent of the medial PFC and NAC. $\boldsymbol{B}, \boldsymbol{C}$, Disconnection of this pathway increased choice latencies $(\boldsymbol{B})$ and trial omissions (C). Sal, Saline; Ipsi, ipsilateral; Disc, disconnection. ${ }^{*} p<0.05$ versus saline.

We exploited these dissociable axonal pathway to selectively disrupt bottom-up and top-down communication between the BLA and PFC. Specifically, by unilaterally targeting ventrolateral amydalofugal axons, combined with contralateral inactivation of the PFC, we selectively disrupted information transfer through the ascending BLA $\rightarrow$ PFC pathway. Conversely, asymmetrical inactivation of ventromedial internal capsule and BLA selectively disrupted information transfer through the descending PFC $\rightarrow$ BLA pathway (Fig. 7).

\section{Effect of functional disconnection of the ascending versus descending BLA-medial PFC pathways on probabilistic discounting}

Initially, 15 rats in the ascending pathway group were trained for $37 \mathrm{~d}$ before showing stable choice behavior and receiving microinfusion test days. Five rats had inaccurate placements in the amydalofugal pathway (mainly in ventrolateral or medial striatum), resulting in a final $n$ of 10 rats in this group. We observed that disconnection of ascending inputs from the BLA to the medial PFC had no effect on choice of the Large/Risky lever (main effect of Treatment: $F_{(2,18)}=0.66, p=$ n.s.; Treatment $\times$ Block interaction: $F_{(6,54)}=1.97$, n.s.; Fig. $\left.8 \mathrm{~A}\right)$. No other performance measures (response latencies, locomotion, omissions) were af- fected by inactivation treatments (all Fs $<$ 1 , all ps > 0.40; Table 1).

For the descending pathway, 16 rats were trained to stability for $36 \mathrm{~d}$ before proceeding to microinfusion test days. One animal died after surgery and four had inaccurate placements outside of the internal capsule, resulting in a final $n$ of 11 rats in this group. In stark contrast to the ascending pathway, disruption of descending inputs from the medial PFC to the BLA significantly increased choice of the Large/Risky lever compared with saline (Fig. $8 B$ ), similar to the effect induced by the overall medial PFC-BLA disconnection. In this experiment, alterations in choice induced by ipsilateral inactivation relative to saline were highly variable across animals, even though these treatments had no overall effect on choice behavior. This variability limited our ability to detect statistically significant effects of Treatment $\left(F_{(2,20)}=2.01, p=0.16\right)$ or a Treatment $\times$ Block interaction $\left(F_{(6,60)}=0.90\right.$, $p=0.50)$ in the overall ANOVA. Nevertheless, targeted comparisons confirmed that asymmetrical inactivations significantly increased choice of the Large/Risky lever relative to saline $\left(F_{(1,10)}=6.80, p=\right.$ $0.025)$, whereas ipsilateral inactivations $\operatorname{did} \operatorname{not}\left(F_{(1,10)}=0.02\right.$, n.s. $)$.

As was observed following the full BLA-PFC disconnection, the increase in choice of the Large/Risky lever was associated with reduced sensitivity to negative feedback. In this instance, asymmetrical inactivations caused a significant reduction in the tendency to choose the safe option on trials following a nonrewarded risky choice $\left(F_{(1,10)}=6.23, p=0.03\right.$; Fig. $8 C)$. However, win-stay tendencies following asymmetrical disconnection did not differ from saline $\left(F_{(1,10)}=0.98\right.$, n.s.; Fig. $\left.8 C\right)$. No other performance measures were affected by inactivations (all Fs $<2.30$, all $p>0.12$; Table 1). Collectively, these data show that regulation of decision making by prefrontal-amygdalar circuitry is achieved primarily by top-down control of the BLA by the medial PFC. When risky options become less profitable over time, descending flow of information from the PFC mitigates bias toward larger rewards driven by BLA-NAc circuitry.

\section{Discussion}

All things being equal, the natural inclination is to go for larger versus smaller payoffs. However, if larger rewards carry some degree of risk/uncertainty, we must integrate information about their magnitude and probability to choose options that may be more profitable in the long-term. These decisions become substantially more complex in dynamic environments where the likelihood of obtaining rewards is volatile. Allocation of resources (as may occur when managing financial investments) requires one to monitor changes in the rate of return of potentially larger, but probabilistic options. If they become too low, it may be more advantageous to reallocate resources to options that yield smaller but more certain returns. The present data suggest that parallel 

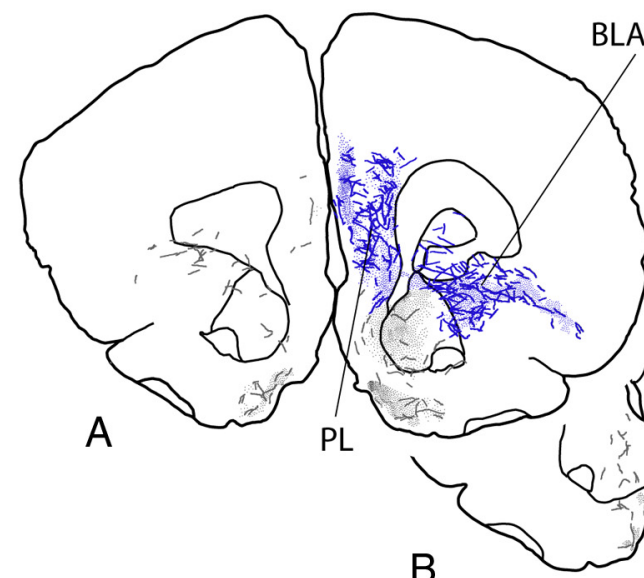

$\mathrm{BLA} \rightarrow \mathrm{PFC}$

B

$\mathrm{Acb}$

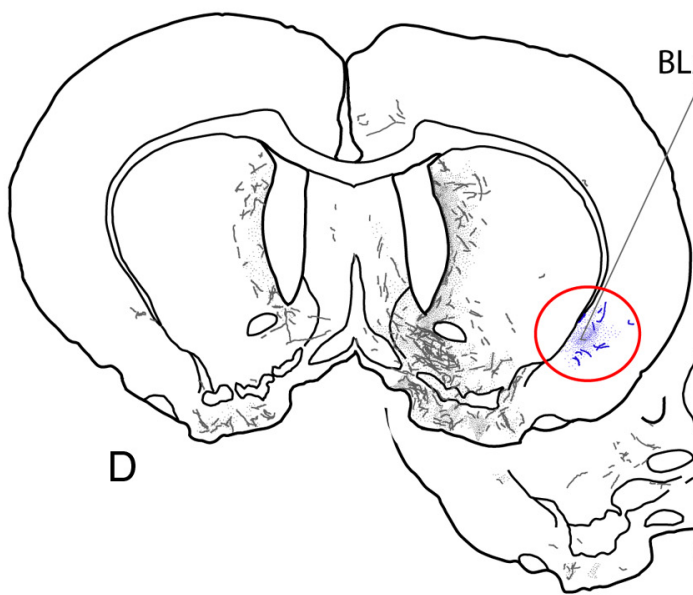

$\mathrm{BLA} \rightarrow \mathrm{PFC}$

E

C

$\mathrm{BLA} \rightarrow \mathrm{PFC}$

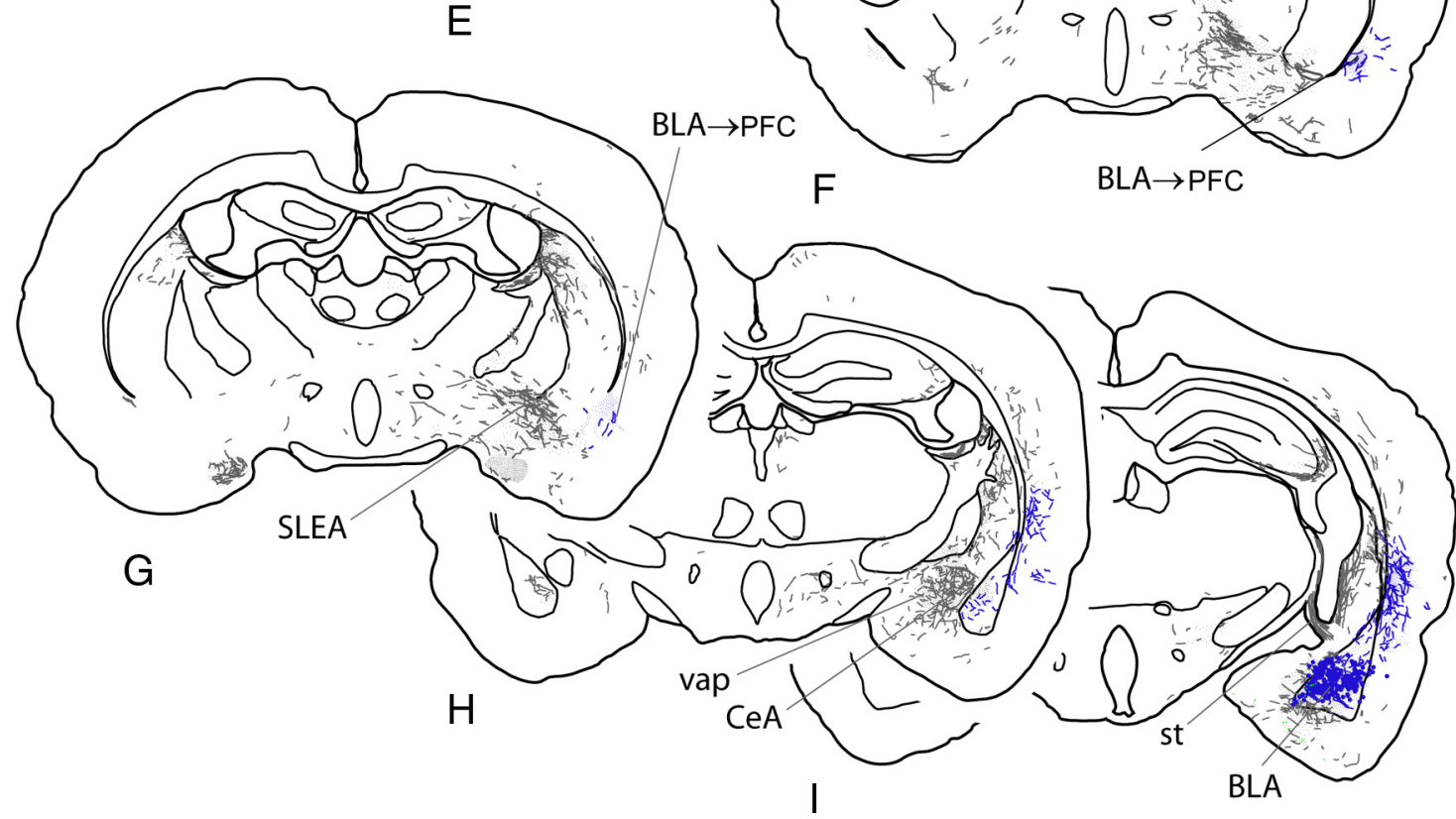

Figure 5. A-I, Diagrams of sections of rat brain ordered from rostral to caudal levels and indicating anterograde labeling produced by an injection of the anterograde tracer biotinylated dextran amine into the BLA, represented by the blue dots in I. Labeled fibers are represented by short lines, which indicate directional orientation, and areas with dense terminal-like labeling are indicated by fine stippling. The pathway from the BLA to the medial prefrontal cortex is highlighted in blue and the site where bupivacaine was infused in the present study to inactivate the pathway is circled in red (D). AC, Anterior cingulate cortex; Acb, nucleus accumbens; BST, bed nucleus of stria terminalis; CeA, central nucleus of the amygdala; $\mathrm{CPu}$, caudate-putamen; ec, external capsule; PL, prelimbic cortex; SLEA, sublenticular extended amygdala; st, stria terminalis; vap, ventral amygdalofugal pathway. 


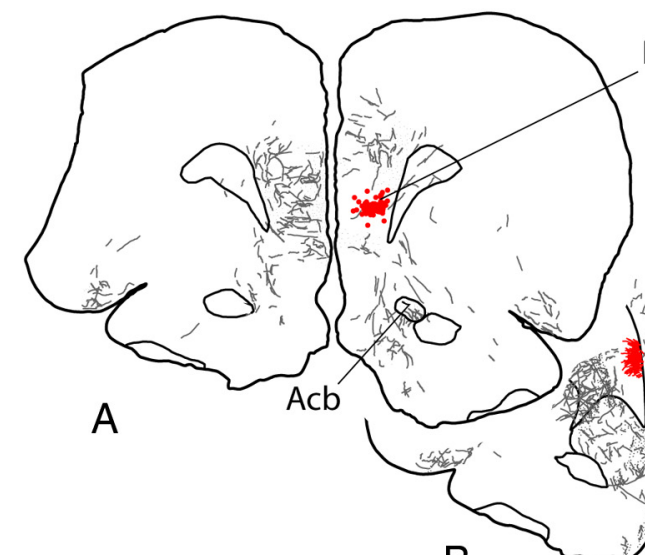

B

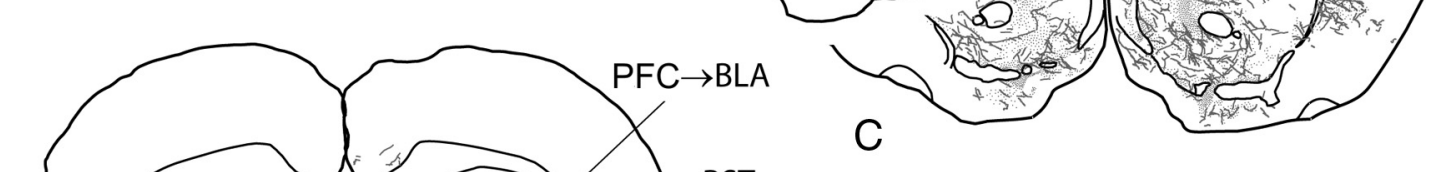

C
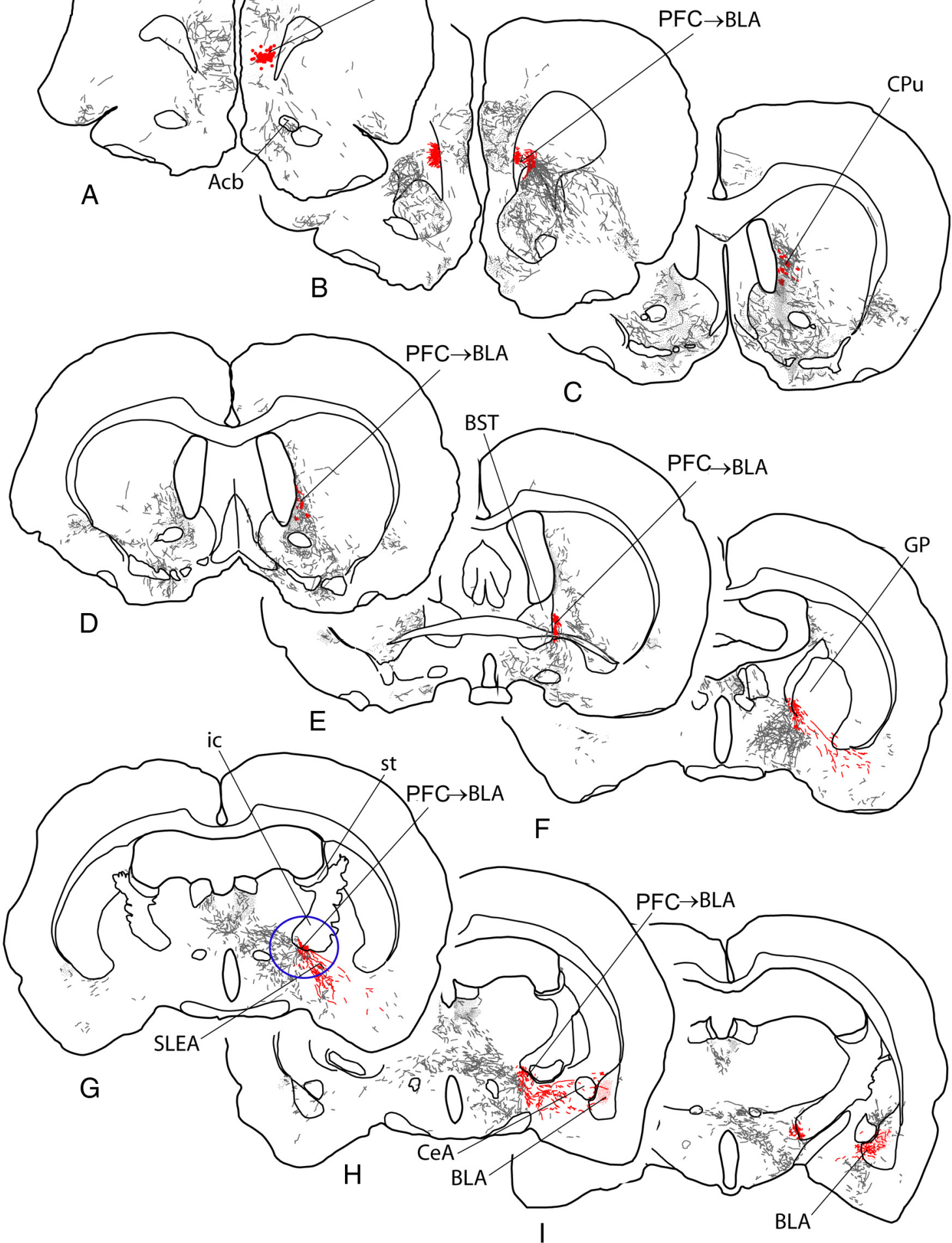

Figure 6. $A-I$, Diagrams of sections of rat brain ordered from rostral to caudal levels and indicating anterograde labeling produced by an injection of the anterograde tracer Phaseolus vulgaris leucoagglutinin into the prelimbic cortex (PL), represented by the red dots in $\boldsymbol{A}$. Labeled fibers are represented by short lines, which indicate directional orientation, and areas with dense terminal-like labeling are indicated by fine stippling. The pathway from the medial PFC to the basolateral amygdala is highlighted in red and the site where bupivacaine was infused in the present study to inactivate the pathway is circled in blue (G). Acb, Nucleus accumbens; BST, bed nucleus of stria terminalis; (eA, central nucleus of the amygdala; (Pu, caudate-putamen; GP, globus pallidus; ic, internal capsule; SLEA, sublenticular extended amygdala; st, stria terminalis. 


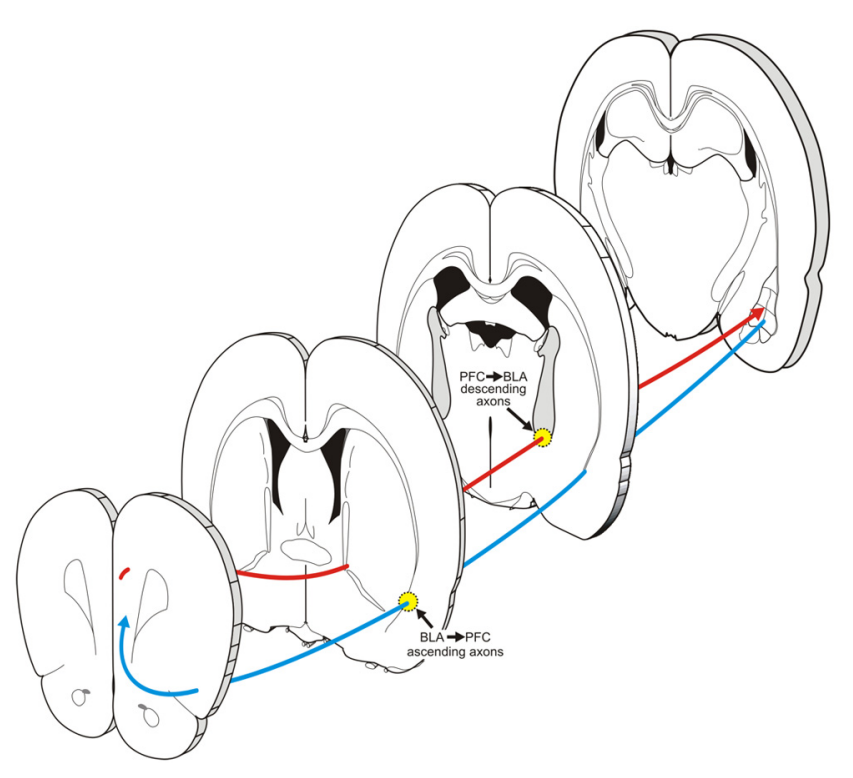

Figure 7. Summary diagram displaying the routes that ascending and descending axons in BLA-PFC pathways take through different sections of the rat brain, highlighting the regions targeted for bupivacaine infusions in disconnection experiments targeting the ascending and descending BLA-PFC pathways.

amygdalar, ventral striatal, and prefrontal circuits make dissociable contributions to choice biases and help regulate whether one continues to pursuing large, uncertain options or choose more conservative, reliable ones.

\section{Subcortical, amygdala-striatal circuitry drives choice toward larger, uncertain rewards}

BLA projections modulate NAc activity and are proposed to influence the direction of behavior toward reward-related stimuli (Everitt et al., 1999; Floresco et al., 2001; Setlow et al., 2002; Ambroggi et al., 2008). Here we show that BLA-NAc disconnections shifted bias away from larger, uncertain rewards, primarily when this option would yield a greater or equal amount of reward than the alternative (i.e.; the first three trial blocks). A decrease in choice of the Large/Risky option was observed after ipsilateral and asymmetrical disconnection of BLA-NAc circuitry, suggesting that disrupting communication between these regions in one hemisphere can be sufficient to alter risky choice (at least in a subset of animals). However, it is unlikely that this effect of ispilateral inactivations was attributable to a mass action effect (i.e., a nonspecific disruption caused by two infusions), because neither ipsilateral nor asymmetrical inactivation of the PFC and NAc affected choice. There have been other reports that ipsilateral inactivation of the BLA and other regions can alter certain behaviors (Lasseter et al., 2011). However, more often than not, ipsilateral inactivations of two brain regions either have a reduced or a lack of effect on other forms of cognition (Chudasama et al., 2003; Dunnett et al., 2005; Block et al., 2007; Maeng et al., 2010). Probabilistic discounting may require greater cognitive processing than simpler behaviors (e.g., conditioning) and may explain why ipsilateral BLANAc disconnections altered behavior in some rats. Nevertheless, the fact that, using a within-subjects design, we observed asymmetrical BLA-NAc disconnections significantly reduced risky choice, even in a subset of animals where ipsilateral disconnections did not, confirms that serial communication between these nuclei biases choice toward larger, uncertain rewards.
Importantly, BLA-NAc disconnections did not affect reward magnitude discrimination, demonstrating that preference for larger rewards is not dependent on serial communication within this circuit. Rather, it appears to make a more selective contribution to decision making when the subjective value of objectively larger rewards is diminished by uncertainty. This is in keeping with a growing literature showing that the BLA and NAc aid in overcoming a variety of costs (e.g., delays, effort) that diminish subjective reward value in the pursuit of objectively larger rewards (Floresco et al., 2008; Ghods-Sharifi et al., 2009; Stopper and Floresco, 2011).

Neurophysiological studies report increased activation of amygdala and NAc correlating with selection of larger or more valuable rewards, even when their receipt is uncertain, suggesting that activity within this circuitry encodes the relative value of different options (Ernst et al., 2004; Kuhnen and Knutson, 2005; Blair et al., 2006; Marsh et al., 2007; Smith et al., 2009). Our data expand on this, suggesting that increased NAc activity associated with choice of larger, riskier rewards is likely driven by excitatory inputs from the BLA. Furthermore, they confirm that the direction of choice is causally linked to increased activation of NAc circuitry, which may influence behavior through descending projections to motor sites (Zahm and Heimer, 1990; Floresco, 2007).

Top-down control of amygdala-NAc circuitry by medial PFC In contrast to BLA-NAc disconnections, disrupting communication between BLA-PFC increased choice of the Large/Risky option. What is striking about this result is that although it closely resembles effects of PFC inactivation under similar task conditions (St. Onge and Floresco, 2010), it is opposite to that induced by BLA inactivation (Ghods-Sharifi et al., 2009). Thus, if these regions were acting independently, a parsimonious expectation would be that disruption of activity in BLA and PFC would cause no net change in choice, with the effect one inactivation effectively cancelling out the other. The fact that disruption of BLA-PFC circuitry induced a prefrontal-like profile suggests that frontal-temporal communication may help track decision outcomes over time and facilitate adjustments in choice biases. In addition, these data highlight the utility of using a circuit analysis approach to understanding the neural basis of decision making, rather than assessing the contribution of different brain regions in isolation.

We next sought to identify whether the effects BLA-PFC disconnections were caused by disrupting bottom-up (BLA-toPFC) or top-down (PFC-to-BLA) signaling. Guided by our neuroanatomical experiments, we were able to selectively target ascending BLA $\rightarrow$ PFC and descending $\mathrm{PFC} \rightarrow$ BLA axonal pathways. Disconnection of the ascending pathway did not produce a reliable change in choice. Thus, even though the BLA may send information about reward value to the PFC, impeding the flow of information in this pathway is not sufficient to alter overt decision making about probabilistic rewards.

In contrast, disconnection of descending $\mathrm{PFC} \rightarrow \mathrm{BLA}$ inputs increased choice of the Large/Risky option in a manner identical to full disconnection of this circuit. This was reflected by a reduced tendency to select the certain option following a nonrewarded risky choice, indicative of diminished negative feedback sensitivity. This finding suggests that $\mathrm{PFC} \rightarrow \mathrm{BLA}$ inputs influence decision biases by providing information about reward omissions that in turn influence direction of subsequent choices. Disruption of this circuit impaired use of information about recent losses to adapt behavior, which may have permitted subcortical circuitry to persist in biasing choice toward the Large/Risky option despite its decreasing profitability over time. The idea that 


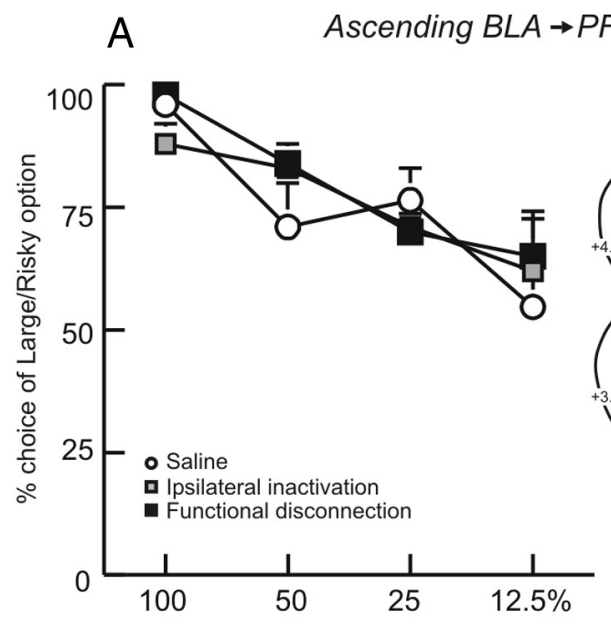

Large/Risky lever probability by block

\section{B}

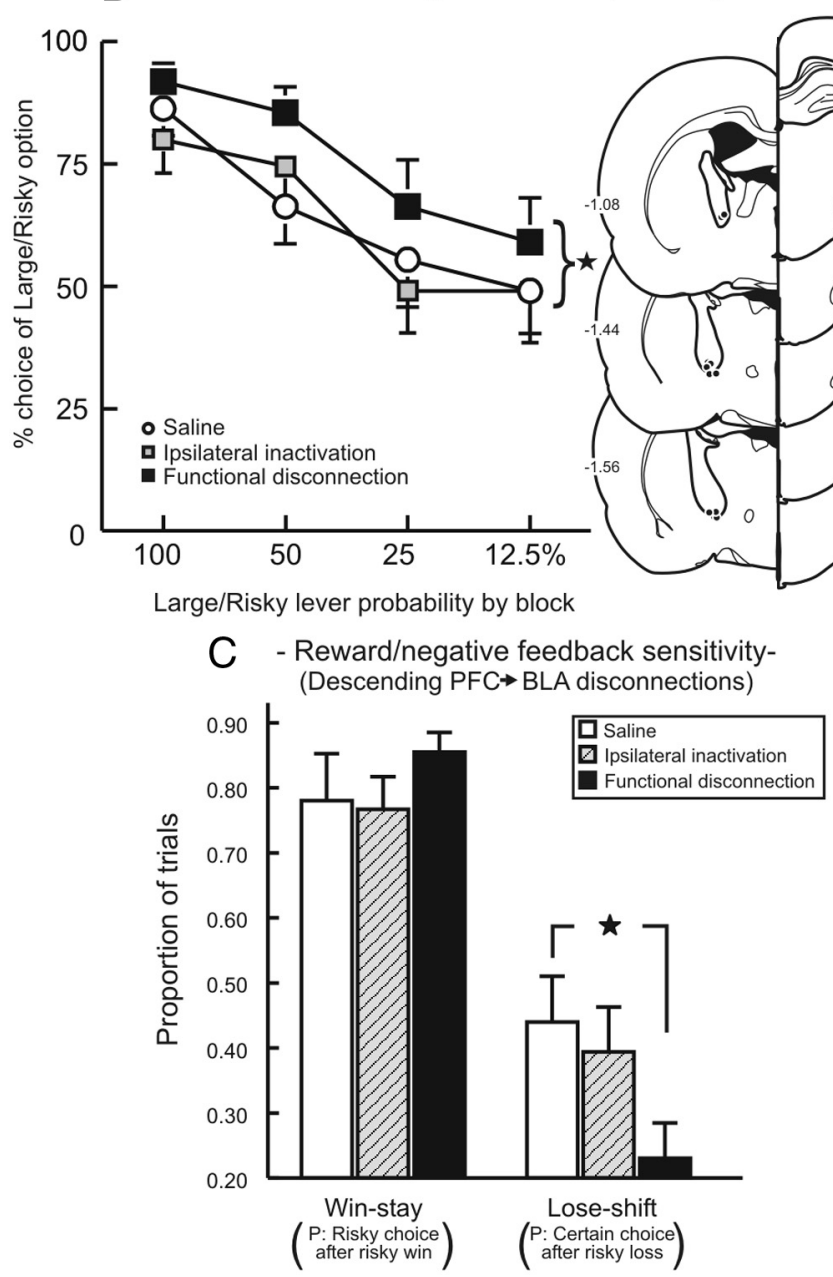

Figure 8. Effect of disconnecting ascending and descending pathways between the medial PFC and BLA on probabilistic discounting. A, Ascending BLA $\rightarrow$ PFC pathway. Left, Choice for the Large/Risky lever following disconnection of the PFC and ventrolateral amydalofugal pathway and control treatments. Disconnection of the ascending pathway had no effect on choice. Right, Acceptable location of infusions in the PFC and ventrolateral edge of the corpus callosum. $\boldsymbol{B}$, Descending PFC $\rightarrow$ BLA pathway. Left, In contrast, disconnection of the descending pathway increased Large/Risky choice. ${ }^{*} p<0.05$, disconnection versus saline. Right, Acceptable location of infusions in BLA and ventromedial internal capsule. C, Win-stay and lose-shift data for descending pathway disconnections. Black star denotes a significant difference from saline, $p<0.05$. Increased risky choice after disconnection of the descending pathway was attributable to a reduced sensitivity to reward omissions (decreased lose-shift performance).
PFC exerts top-down control over subcortical regions in not new, although this conclusion has been derived primarily from indirect evidence from lesion and imaging studies (Etkin et al., 2006; Wager et al., 2008). To our knowledge, this is the first use of a combined neuroanatomical/disconnection approach to dissect directionality of communication within these circuits and directly demonstrate top-down control of the amygdala by PFC.

PFC projections to the ventral striatum are critical for various executive functions, including attention, working memory, cognitive flexibility, and effort-related decision making (Floresco et al., 1999; Christakou et al., 2004; Block et al., 2007; Hauber and Sommer, 2009). As such, it was surprising that disrupting this corticostriatal pathway did not affect probabilistic discounting, suggesting that these two regions work relatively independently in guiding decision biases. Nevertheless, this manipulation did disrupt other aspects of task performance, increasing choice latencies and reducing the number of choices made. Collectively, these findings reveal that parallel output pathways from the frontal lobes to separate subcortical targets regulate distinct aspects of decision making in a dissociable manner. Projections to BLA aid in adjusting choice biases, whereas those to the NAc enable staying on task and facilitate the relative speed of response selection, ensuring decisions are made in a timely fashion.

Medial regions of the PFC, including the anterior cingulate, have been associated with cognitive control and monitoring changes in reward contingencies (Botvinick et al., 2001; Rogers et al., 2004; Marsh et al., 2007). Progressive increases in anterior cingulate activity are associated with disengagement from options providing diminishing returns in favor of exploring novel reward sources (Hayden et al., 2011). Thus, the anterior cingulate may contribute to learning about rewarded/nonrewarded actions over time to determine which options are relatively more profitable, which in turn may influence whether decision biases promote exploitation of currently profitable situations or lean more toward exploration of new ones when rewards become less frequent (Daw et al., 2006; Kennerley et al., 2006; Rushworth, 2008). This notion is consistent with neurophysiological studies implicating this region in "marshaling neural resources so that animals can learn when reward contingencies change" (Bryden et al., 2011). Furthermore, detection of changes in reward contingencies have been proposed to be mediated by interactions between anterior cingulate-amygdala circuitry (Roesch 
et al., 2010; Bryden et al., 2011), an idea entirely consistent with our findings.

Our data provide further insight into the dynamic interactions between prefrontal, amygdalar, and striatal systems that shape decision biases. In situations where reward probabilities are volatile, subcortical circuits linking the BLA and NAc provide a visceral, intuitive bias toward options that may yield larger rewards. In comparison, the PFC appears to play a supervisory role, monitoring frequency of rewarded/nonrewarded actions over time. As reward omissions accumulate, indicating that larger, riskier reward options are becoming less profitable, the PFC aids in learning about changes in reward contingencies, which may facilitate shifts in decision biases. Alterations in decision biases are executed via descending pathways from PFC to BLA, which temper the influence that BLA-NAc circuitry exerts over the direction of choice, potentially via feedforward inhibition within this pathway (Rosenkranz and Grace, 2002). This latter notion is in keeping with studies transcranial magnetic stimulation in humans that support the idea that the PFC can override decision-making biases mediated by other brain systems (Knoch et al., 2006; Figner et al., 2010). Thus, our findings shed new light on the dynamic competition between prefrontal, amygdalar, and ventral striatal circuits and how their interplay shape decision biases and underlies the struggles we face when evaluating choices that vary in terms of potential risks and rewards.

\section{References}

Ambroggi F, Ishikawa A, Fields HL, Nicola SM (2008) Basolateral amygdala neurons facilitate reward-seeking behavior by exciting nucleus accumbens neurons. Neuron 59:648-661.

Bacon SJ, Headlam AJ, Gabbott PL, Smith AD (1996) Amygdala input to medial prefrontal cortex (mPFC) in the rat: A light and electron microscope study. Brain Res 720:211-219.

Balleine BW, Killcross S (2006) Parallel incentive processing: an integrated view of amygdala function. Trends Neurosci 29:272-279.

Bezzina G, Body S, Cheung TH, Hampson CL, Bradshaw CM, Szabadi E, Anderson IM, Deakin JF (2008) Effect of disconnecting the orbitofrontal prefrontal cortex from the nucleus accumbens core on inter-temporal choice behaviour: a quantitative analysis. Behav Brain Res 191:272-279.

Blair K, Marsh AA, Morton J, Vythilingam M, Jones M, Mondillo K, Pine DC, Drevets WC, Blair JR (2006) Choosing the lesser of two evils, the better of two goods: specifying the roles of ventromedial prefrontal cortex and dorsal anterior cingulate in object choice. J Neurosci 26:11379-11386.

Block AE, Dhanji H, Thompson-Tardif SF, Floresco SB (2007) Thalamicprefrontal cortical-ventral striatal circuitry mediates dissociable components of strategy set shifting. Cereb Cortex 17:1625-1636.

Botvinick MM, Braver TS, Barch DM, Carter CS, Cohen JD (2001) Conflict monitoring and cognitive control. Psychol Rev 108:624-652.

Brog JS, Salyapongse A, Deutch AY, Zahm DS (1993) The patterns of afferent innervation of the core and shell in the "accumbens" part of the rat ventral striatum: immunohistochemical detection of retrogradely transported fluoro-gold. J Comp Neurol 338:255-278.

Bryden DW, Johnson EE, Tobia SC, Kashtelyan V, Roesch MR (2011) Attention for learning signals in anterior cingulate cortex. J Neurosci 31:18266-18274.

Christakou A, Robbins TW, Everitt BJ (2004) Prefrontal cortical-ventral striatal interactions involved in affective modulation of attentional performance: implications for corticostriatal circuit function. J Neurosci 24:773-780.

Chudasama Y, Baunez C, Robbins TW (2003) Functional disconnection of the medial prefrontal cortex and subthalamic nucleus in attentional performance: evidence for corticosubthalamic interaction. J Neurosci 23:5477-5485.

Cohen MX, Heller AS, Ranganath C (2005) Functional connectivity with anterior cingulate and orbitofrontal cortices during decision-making. Brain Res Cogn Brain Res 23:61-70.

Daw ND, O’Doherty JP, Dayan P, Seymour B, Dolan RJ (2006) Cortical substrates for exploratory decisions in humans. Nature 441:876-879.
Dunnett SB, Meldrum A, Muir JL (2005) Frontal-striatal disconnection disrupts cognitive performance of the frontal-type in the rat. Neuroscience 135:1055-1065.

Ernst M, Nelson EE, McClure EB, Monk CS, Munson S, Eshel N, Zarahn E, Leibenluft E, Zametkin A, Towbin K, Blair J, Charney D, Pine DS (2004) Choice selection and reward anticipation: an fMRI study. Neuropsychologia 42:1585-1597.

Etkin A, Egner T, Peraza DM, Kandel ER, Hirsch J (2006) Resolving emotional conflict: a role for the rostral anterior cingulate cortex in modulating activity in the amygdala. Neuron 51:871-882.

Everitt BJ, Parkinson JA, Olmstead MC, Arroyo M, Robledo P, Robbins TW (1999) Associative processes in addiction and reward: the role of amygdala-ventral striatal subsystems. Ann N Y Acad Sci 877:412-438.

Figner B, Knoch D, Johnson EJ, Krosch AR, Lisanby SH, Fehr E, Weber EU (2010) Lateral prefrontal cortex and self-control in intertemporal choice. Nat Neurosci 13:538-539.

Floresco SB (2007) Dopaminergic regulation of limbic-striatal interplay. J Psychiatry Neurosci 32:400-411.

Floresco SB, Braaksma DN, Phillips AG (1999) Thalamic-cortical-striatal circuitry subserves working memory during delayed responding on a radial arm maze. J Neurosci 19:11061-11071.

Floresco SB, Blaha CD, Yang CR, Phillips AG (2001) Dopamine D1 and NMDA receptors mediate potentiation of basolateral amygdala-evoked firing of nucleus accumbens neurons. J Neurosci 21:6370-6376.

Floresco SB, St. Onge JR, Ghods-Sharifi S, Winstanley CA (2008) Corticolimbic-striatal circuits subserving different forms of cost-benefit decision making. Cogn Affect Behav Neurosci 8:375-389.

Ghods-Sharifi S, St. Onge JR, Floresco SB (2009) Fundamental contribution by the basolateral amygdala to different forms of decision making. J Neurosci 29:5251-5259.

Groenewegen HJ, Berendse HW, Wolters JG, Lohman AH (1990) The anatomical relationship of the prefrontal cortex with the striatopallidal system, the thalamus, and the amygdala: evidence for a parallel organization. Prog Brain Res 85:95-116.

Hampton AN, Adolphs R, Tyszka MJ, O’Doherty JP (2007) Contributions of the amygdala to reward expectancy and choice signals in human prefrontal cortex. Neuron 55:545-555.

Hauber W, Sommer S (2009) Prefrontostriatal circuitry regulates effortrelated decision making. Cereb Cortex 19:2240-2247.

Hayden BY, Heilbronner SR, Pearson JM, Platt ML (2011) Surprise signals in anterior cingulate cortex: neuronal encoding of unsigned reward prediction errors driving adjustment in behavior. J Neurosci 31:4178-4187.

Kennerley SW, Walton ME, Behrens TE, Buckley MJ, Rushworth MF (2006) Optimal decision making and the anterior cingulate cortex. Nat Neurosci 9:940-947.

Knoch D, Gianotti LR, Pascual-Leone A, Treyer V, Regard M, Hohmann M, Brugger P (2006) Disruption of right prefrontal cortex by lowfrequency repetitive transcranial magnetic stimulation induces risktaking behavior. J Neurosci 26:6469-6472.

Knutson B, Taylor J, Kaufman M, Peterson R, Glover G (2005) Distributed neural representation of expected value. J Neurosci 25:4806-4812.

Krettek JE, Price JL (1977) Projections from the amygdaloid complex to the cerebral cortex and thalamus in the rat and cat. J Comp Neurol 172:687-722.

Kuhnen CM, Knutson B (2005) The neural basis of financial risk taking. Neuron 47:763-770.

Lasseter HC, Wells AM, Xie X, Fuchs RA (2011) Interaction of the basolateral amygdala and orbitofrontal cortex is critical for drug context-induced reinstatement of cocaine-seeking behavior in rats. Neuropsychopharmacology 36:711-720.

Maeng LY, Waddell J, Shors TJ (2010) The prefrontal cortex communicates with the amygdala to impair learning after acute stress in females but not males. J Neurosci 30:16188-16196.

Marsh AA, Blair KS, Vythilingam M, Busis S, Blair RJ (2007) Response options and expectations of reward in decision-making: the differential roles of dorsal and rostral anterior cingulate cortex. Neuroimage 35:979-988.

McDonald AJ (1987) Organization of amygdaloid projections to the mediodorsal thalamus and prefrontal cortex: A fluorescence retrograde transport study in the rat. J Comp Neurol 262:46-58.

McDonald AJ (1991a) Organization of amygdaloid projections to the prefrontal cortex and associated striatum in the rat. Neuroscience 44:1-14.

McDonald AJ (1991b) Topographical organization of amygdaloid projec- 
tions to the caudatoputamen, nucleus accumbens, and related striatal-like areas of the brain. Neuroscience 44:15-33.

McDonald AJ, Mascagni F, Guo L (1996) Projections of the medial and lateral prefrontal cortices to the amygdala: a Phaseolus vulgaris leucoagglutinin study in the rat. Neuroscience 71:55-75.

Orozco-Cabal L, Pollandt S, Liu J, Vergara L, Shinnick-Gallagher P, Gallagher JP (2006) A novel rat medial prefrontal cortical slice preparation to investigate synaptic transmission from amygdala to layer $\mathrm{V}$ prelimbic pyramidal neurons. J Neurosci Methods 151:148-158.

Paxinos G and Watson C (1998) The rat brain in stereotaxic coordinates, 4th edition. San Diego: Academic.

Pessoa L (2010) Emotion and cognition and the amygdala: from "what is it?" to "what's to be done?" Neuropsychologia 48:3416-3429.

Roesch MR, Singh T, Brown PL, Mullins SE, Schoenbaum G (2009) Ventral striatal neurons encode the value of the chosen action in rats deciding between differently delayed or sized rewards. J Neurosci 29:13365-13376.

Roesch MR, Calu DJ, Esber GR, Schoenbaum G (2010) Neural correlates of variations in event processing during learning in basolateral amygdala. J Neurosci 30:2464-2471.

Rogers RD, Ramnani N, Mackay C, Wilson JL, Jezzard P, Carter CS, Smith SM (2004) Distinct portions of anterior cingulate cortex and medial prefrontal cortex are activated by reward processing in separable phases of decision-making cognition. Biol Psychiatry 55:594-602.

Rosenkranz JA, Grace AA (2002) Cellular mechanisms of infralimbic and prelimbic prefrontal cortical inhibition and dopaminergic modulation of basolateral amygdala neurons in vivo. J Neurosci 22:324-337.

Rushworth MF (2008) Intention, choice, and the medial frontal cortex. Ann N Y Acad Sci 1124:181-207.
Sesack SR, Deutch AY, Roth RH, Bunney BS (1989) Topographical organization of the efferent projections of the medial prefrontal cortex in the rat: an anterograde tract-tracing study with Phaseolus vulgaris leucoagglutinin. J Comp Neurol 290:213-242.

Setlow B, Holland PC, Gallagher M (2002) Disconnection of the basolateral amygdala complex and nucleus accumbens impairs appetitive pavlovian second-order conditioned responses. Behav Neurosci 116:267-275.

Smith BW, Mitchell DG, Hardin MG, Jazbec S, Fridberg D, Blair RJ, Ernst M (2009) Neural substrates of reward magnitude, probability, and risk during a wheel of fortune decision-making task. Neuroimage 44:600-609.

St. Onge JR, Floresco SB (2009) Dopaminergic modulation of risk-based decision making. Neuropsychopharmacology 34:681-697.

St. Onge JR, Floresco SB (2010) Prefrontal cortical contribution to riskbased decision making. Cereb Cortex 20:1816-1828.

St. Onge JR, Abhari H, Floresco SB (2011) Dissociable contributions by prefrontal D1 and D2 receptors to risk-based decision making. J Neurosci 31:8625-8633.

Stopper CM, Floresco SB (2011) Contributions of the nucleus accumbens and its subregions to different aspects of risk-based decision making. Cogn Affect Behav Neurosci 11:97-112.

Tehovnik EJ, Sommer MA (1997) Effective spread and timecourse of neural inactivation caused by lidocaine injection in monkey cerebral cortex. J Neurosci Methods 74:17-26.

Wager TD, Davidson ML, Hughes BL, Lindquist MA, Ochsner KN (2008) Prefrontal-subcortical pathways mediating successful emotion regulation. Neuron 59:1037-1050.

Zahm DS, Heimer L (1990) Two transpallidal pathways originating in the nucleus accumbens. J Comp Neurol 302:437-446. 